\title{
Experiencia en la utilización de hipolipemiantes en una cohorte de pacientes en 12 ciudades colombianas
}

\author{
Juan José Diaztagle* \\ Carlos Alberto Castro** \\ Diana Carolina Buitrago***
}

\begin{abstract}
* Médico internista. Fisiólogo. Epidemiólogo. Profesor. Departamento de fisiología. Universidad Nacional de Colombia. Bogotá D.C. Colombia.
** Médico epidemiólogo. Soluciones Integrales para la Investigación y educación en salud - SIIES Consultores. Profesor asistente. Fundación Universitaria de Ciencias de la Salud. Bogotá D.C. Colombia.

*** Enfermera epidemióloga. Soluciones Integrales para la Investigación y educación en salud - SIIES Consultores. Profesora de cátedra. Fundación Universitaria de Ciencias de la Salud. Bogotá D.C. Colombia.

Correspondencia: Dr. Diaztagle. Dirección: Calle 106 No. 54-15 of. 602. Edificio Grupo7 Torre 8. Bogotá D.C. Colombia. Correo electrónico: juanchotagle@yahoo.es
\end{abstract}

\section{Resumen}

Introducción: Los estudios de utilización de medicamentos sirven para evaluar la efectividad y seguridad de los fármacos en la práctica real, diferente al contexto del estudio clínico controlado. Los hipolipemiantes actúan sobre el perfil lipídico disminuyendo el riesgo de enfermedades cardiovasculares. Objetivo: Describir el desempeño clínico y seguridad de la utilización de medicamentos hipolipemiantes en la práctica médica real en una cohorte de pacientes con diagnóstico de dislipidemia. Metodología: Estudio observacional de cohorte. Se siguió una cohorte de pacientes con indicación de hipolipemiantes durante 6 meses, en 12 ciudades de Colombia pertenecientes a un registro biomédico de seguimiento de pacientes tratados con medicamentos del portafolio de Abbott. Se midieron variables demográficas y clínicas basales, de seguridad y de desempeño clínico de los medicamentos sobre el perfil lipídico a los 3 y 6 meses. Resultados: Se siguieron 501 pacientes en tratamiento con hipolipemiantes. Las estatinas solas disminuyeron el colesterol de baja densidad de $249 \mathrm{mg} /$ dL $(R I Q=226-268)$ en la medición basal a 190 (177.6-210) y 170 (108-170) en la segunda y tercera medición, respectivamente. Para estatina + ezetimibe, de $167 \mathrm{mg} / \mathrm{dL}(\mathrm{RIQ}=139-184$ ) a 132 (110-150) y 128.5 (101.5-128.5). El fenofibrato disminuyó los triglicéridos de 275 mg/dL (RIQ=219346) a 201 (172-239) y 150.5 (140-150.5). Conclusiones: la administración de estatinas sola o en combinación disminuyó los niveles de LDL y colesterol total, mientras que el fenofibrato demostró su efectividad al disminuir los triglicéridos. No se reportaron efectos adversos. Hubo una adherencia parcial del médico tratante a la guía de práctica clínica para dislipidemias. MÉD.UIS.2019;32(1):13-20.

Palabras claves: Farmacovigilancia. Hipolipemiantes. Colesterol. Registros médicos. Dislipidemia.

\section{Experience in the use of oral lipid-lowering medicines in a group of patients followed in 12 colombian cities}

\begin{abstract}
introduction: Drug use studies are important to evaluate the effectiveness and safety of drugs in daily practice, outside the controlled clinical study. Lipid-lowering drugs act on the lipid profile, decreasing the risk of cardiovascular diseases. Objective: To describe the clinical performance and safety of the use of lipid-lowering drugs in real practice in a group of patients diagnosed with dyslipidemia. Methods: An observational, descriptive cohort study. A cohort of patients with hypolipidemic indication for 6 months was followed in 12 cities of Colombia that belong to the biomedical registry of follow-up of patients treated with medicines from the Abbott portfolio. Baseline demographic and clinical variables, safety and efectivity of the drugs were measured on the lipid profile at 3 and 6 months. Results: 501 patients received lipid-lowering agents. Statins alone decreased the low density (LDL) cholesterol of $249 \mathrm{mg} / \mathrm{dL}(\mathrm{RIQ}=226-268)$ at
\end{abstract}

Artículo recibido el 10 de mayo del 2018 y aceptado para publicación el 28 de enero del 2019 
baseline to 190 (177.6-210) and 170 (108-170) at the second and third measurements, respectively. For statin + ezetimibe, from $167 \mathrm{mg} / \mathrm{dL}$ $(\mathrm{RIQ}=139-184)$ to 132 (110-150) and 128.5 (101.5-128.5). Fenofibrate decreased triglycerides from $275 \mathrm{mg} / \mathrm{dL}(\mathrm{RIQ}=219-346)$ to 201 (172-239) and 150.5 (140-150.5). Conclusions: The administration of statins alone or in combination decreased LDL and total cholesterol levels, while fenofibrate demonstrated its effectiveness in lowering triglycerides. No adverse effects were reported. There was partial adherence of the treating physician to GPC for dyslipidemias. There were no adverse events. MÉD.UIS.2019;32(1):13-20.

Keywords: Pharmacovigilance. Hypolipidemic Agents. Cholesterol. Registries. Dyslipidemia.

¿Cómo citar este artículo?: Diaztagle JJ, Castro CA, Buitrago DC. Experiencia en la utilización de hipolipemiantes en una cohorte de pacientes en 12 ciudades colombianas. MÉD.UIS.2019;32(1):1320. doi: 10.18273/revmed.v32n1-2019002

\section{Introducción}

La investigación sobre la utilización de medicamentos en la práctica clínica diaria es importante en la actualidad ya que permite conocer lo que sucede con la administración de un medicamento en los pacientes en condiciones de vida real'. Los estudios sobre utilización de medicamentos fueron definidos a finales de los años 70 por la Organización Mundial de la Salud(OMS) como estudios dedicados al mercadeo, distribución, prescripción y uso de medicamentos en la sociedad como parte de la vida real con énfasis en las consecuencias médicas, sociales y económicas de su utilización. Dentro de este concepto, uno de sus objetivos específicos es evaluar los resultados y consecuencias de la utilización de medicamentos ${ }^{2}$. Dos aspectos fundamentales se encuentran relacionados con este último punto; la efectividad y la seguridad.

Los medicamentos son aprobados por las entidades competentes con estudios de fase III (estudios de eficacia) en donde se evalúan los medicamentos en condiciones controladas, como parte de investigaciones donde hay criterios bien definidos de selección de pacientes, seguimiento estricto de los mismos, y en muchas ocasiones, realizados en centros hospitalarios de atención especializada. Estas condiciones distan de la utilización del medicamento en la práctica diaria. En este sentido se hacen necesarios los estudios de desempeño clínico, los cuales evalúan el medicamento en condiciones del ejercicio cotidiano de la práctica médica ${ }^{3,4}$.

Además de la efectividad, la seguridad es otro aspecto importante en el uso de los medicamentos'. Este punto también debe ser evaluado en la práctica diaria y hace parte del concepto de farmacovigilancia, la cual se define según la OMS como la disciplina encargada de la detección, evaluación, entendimiento y prevención de los efectos adversos relacionados con medicamentos 5 . La vigilancia de los medicamentos posterior a su comercialización es fundamental teniendo en cuenta que en ocasiones se pueden detectar eventos adversos relacionados con los medicamentos no identificables en estudios previos a la aprobación y que en algunos casos han llevado a las agencias competentes a retirar medicamentos aprobados o realizar advertencias en su utilización ${ }^{6-9}$. Para este caso, los medicamentos hipolipemiantes son la principal línea de tratamiento para la dislipidemia, la cual es considerada como un factor de riesgo para enfermedades cardiovasculares. De hecho, se ha documentado que el LDL explica cerca de dos tercios del riesgo poblacional atribuible para enfermedad cerebrovascular ${ }^{10-12}$. La OMS para el 2011 estimó que 2,6 millones de fallecimientos a nivel mundial se relacionaron directamente a enfermedades como la dislipidemia, y teniendo en cuenta la prevalencia mundial del $39 \%$ es importante definir estrategias preventivas para disminuir estos indicadores ${ }^{13,14}$. En este contexto existe una alta evidencia científica que soporta el uso de las estatinas para la prevención de la enfermedad cardiovascular aterotrombótica, como prevención secundaria y en algunos pacientes también para prevención primaria. Estos medicamentos han demostrado su beneficio en regímenes de alta y moderada intensidad en donde reducen el colesterol LDL en valores mayores al 50\% o entre 30 y $50 \%$ respectivamente y en un espectro de colesterol LDL basal $\geq 70 \mathrm{mg} / \mathrm{dL}^{15}$.

Aunque se encuentra ampliamente demostrada su utilidad, desafortunadamente su utilización no es adecuada en la práctica clínica. El estudio PURE demostró que la utilización en países de altos ingresos alcanza apenas el $64 \%$ mientras que para América del Sur la situación es muy preocupante con apenas el $14 \%$ de utilización ${ }^{16}$. Algunos estudios realizados en Colombia han mostrado que la situación dista de ser óptima; en un hospital de nivel tres se demostró que 
apenas un $57,7 \%$ de los pacientes con indicaciones recibían estatinas y las metas de colesterol LDL se alcanzaron en el $57,3 \%$ de los casos, mientras que en otro estudio entre 23,5 y $57,2 \%$ de los pacientes catalogados de alto riesgo mantenían metas adecuadas de LDL, y en $14 \%$ no estaba prescrito ${ }^{17,18}$. El objetivo del presente estudio, fue describir el desempeño clínico y la seguridad de la utilización de medicamentos hipolipemiantes en la práctica médica real, en una cohorte de pacientes con diagnóstico de dislipidemia y con indicación de su uso, a partir del registro de seguimiento.

\section{Materiales y métodos}

Se realizó un estudio observacional de cohorte, en el que se siguió un grupo de pacientes, con diagnóstico de dislipidemia, quienes tenían indicación según el médico tratante para iniciar tratamiento farmacológico. Se incluyeron pacientes de doce ciudades de Colombia (Bogotá, San Juan del Cesar, Manizales, Florida Blanca, Bucaramanga, Medellín, Florida, Palmira, Cali, Santa Marta, Barranquilla y Cartagena) quienes fueron atendidos en la consulta privada por un grupo de médicos generales, que decidieron participar voluntariamente y fueron incluidos al estudio de manera consecutiva no probabilística. La recolección de la información se hizo durante seis meses durante el 2016. Se incluyeron pacientes mayores de 18 años, con indicación de tratamiento farmacológico y que no tuvieran contraindicaciones farmacológicas para el uso de estatinas, ezetimibe o fibratos, tales como antecedentes de enfermedad hepática crónica y miopatía de cualquier causa. Se excluyeron los registros que no tuvieran la información completa de antecedentes médicos, registro de la prescripción de los medicamentos y resultados del perfil lipídico. Los pacientes fueron informados del estudio, incluidos voluntariamente y aprobaron la utilización de la información para fines de publicación.

Se recolectaron variables demográficas y se midieron los valores del colesterol total, triglicéridos, colesterol de alta densidad o high density lipoprotein (HDL) y LDL por sus siglas en inglés low-density lipoprotein, antes del inicio del medicamento (basal), y posteriormente se realizaron dos mediciones de control. Los resultados entre las mediciones del perfil lipídico tuvieron un intervalo de tiempo aproximado de 60 a 90 días, en el control uno y control dos con respecto a la vinculación. Las muestras fueron procesadas en los laboratorios de cada ciudad, con certificación vigente según la Secretaria de Salud respectiva. Adicionalmente, se evaluó la frecuencia de eventos adversos por interrogatorio clínico, examen físico y auto reporte.

Para la recolección de la información, se implementó una plataforma remota de captura de la información, teniendo en cuenta el programa "Registro biomédico de seguimiento a la atención médica y a los desenlaces clínicos de pacientes tratados con medicamentos del portafolio de Abbott (RBDC)", el cual se estructuró para determinar el seguimiento y la utilización de medicamentos en enfermedades prevalentes como parte del programa de farmacovigilancia. Se categorizaron los medicamentos de acuerdo con las combinaciones: medicamento solo y en asociación con otras moléculas. También se tuvo en cuenta las familias de los medicamentos para su análisis.

Los médicos fueron capacitados en el manejo de la plataforma, recolección de la información y buenas prácticas clínicas. Una auxiliar entrenada "data entry" realizó visitas mensuales, durante seis meses, para validar la información de los registros en la plataforma contra la fuente original de la información, de acuerdo a una selección aleatoria del 10\% de los registros de cada médico participante. Este estudio fue aprobado por un comité de ética de investigación con seres humanos de la Fundación Universitaria de Ciencias de la Salud.

\section{Análisis estadístico}

Para el análisis se describieron las variables de interés de acuerdo con su escala de medición. Las variables cualitativas (sexo, tipo de medicamento, asociación de fármacos y familias según su composición farmacológica) se presentaron con frecuencias absolutas y relativas. Las variables cuantitativas (edad, colesterol total-CT, triglicéridos-TGC, LDL y $\mathrm{HDL}$ ) se presentaron con medidas de tendencia central y de dispersión, según la distribución de los datos, la cual fue explorada con la prueba estadística de Shapiro Wilk. Se exploraron diferencias entre los resultados del perfil lipídico basal y los del segundo control utilizando la prueba estadística de Wilcoxon. Para determinar diferencias desde el punto de vista estadístico se tuvo en cuenta un valor $\mathrm{p}$ de 0.05 . El análisis de la información se realizó en STATA 12.

\section{Resultados}

Se analizaron 501 pacientes con prescripciones de hipolipemiantes, de los cuales 375 (74.8\%) 
fueron evaluados en el primer control y de estos, 198 pacientes (52.8\%) en el segundo control. Las características generales de la población se muestran en la tabla(Ver Tabla 1).

Tabla 1. Características generales de la población $(n=501)$.

\begin{tabular}{|c|c|}
\hline Edad años - Mediana (RIQ†) & $56(48-67)$ \\
\hline \multicolumn{2}{|c|}{ Sexo $n(\%)$} \\
\hline Femenino & $312(62.3)$ \\
\hline Masculino & $189(37.7)$ \\
\hline \multicolumn{2}{|c|}{ Antecedentes patológicos $+\mathrm{n}(\%)$} \\
\hline Hipertensión arterial & $293(64.4)$ \\
\hline Hipotiroidismo & $12(2.4)$ \\
\hline Artrosis & $7(1.4)$ \\
\hline Gastritis crónica & $6(1.2)$ \\
\hline Patología psiquiátricos & $3(0.6)$ \\
\hline Asma & $2(0.4)$ \\
\hline \multicolumn{2}{|c|}{ Medicamentos n (\%) } \\
\hline Rosuvastatina & $153(30.5)$ \\
\hline Ezetimibe-atorvastatina & $104(20.7)$ \\
\hline Fenofibrato & $99(19.7)$ \\
\hline Rosuvastatina-ezetimibe & $77(15.3)$ \\
\hline Rosuvastatina-fenofibrato & $68(13.5)$ \\
\hline \multicolumn{2}{|l|}{ Combinaciones n (\%) } \\
\hline Estatina + ezetimibe & $181(72.7)$ \\
\hline Estatinas + fibratos & $68(27.3)$ \\
\hline \multicolumn{2}{|c|}{ Monoterapia n (\%) } \\
\hline Estatina & $153(60.7)$ \\
\hline Fibrato & 99 (39.3) \\
\hline
\end{tabular}

† Rango intercuartílico, † n=323 (64.4\%).

Fuente: Autores.

Se compararon los valores de colesterol total, triglicéridos, LDL y HDL al momento de la vinculación y en los controles uno y dos (Ver Tabla 2). En esta tabla, la reducción del LDL, entre la vinculación (To) y el control dos (T2), mostró una reducción de $41 \mathrm{mg} / \mathrm{dl}$ con el uso de estatinas en monoterapia, $40 \mathrm{mg} / \mathrm{dl}$ en Estatinas + Fibrato y $39 \mathrm{mg} / \mathrm{dl}$ para estatinas + ezetimibe. En 148 pacientes (29.5\%) se inició tratamiento con fibrato en monoterapia o combinación con estatinas, los cuales tenían valores de TGC por debajo de $500 \mathrm{mg} / \mathrm{dl}$. En relación con los TGC, ningún paciente evidenció resultados mayores a $500 \mathrm{mg} / \mathrm{dl}$, por lo cual el inicio de fibratos solo mostró una reducción de $125 \mathrm{mg} / \mathrm{dl}$, mientras que la combinación de fibrato + estatinas se observó una reducción de $110 \mathrm{mg} / \mathrm{dl}$.
El análisis del perfil lipídico (CT, TGC, LDL y HDL) de acuerdo con el tratamiento de monoterapia vs. terapia combinada, teniendo en cuenta los valores de la vinculación de los pacientes comparados con el segundo control, se detallan en la Figura 1 (Ver Figura 1).

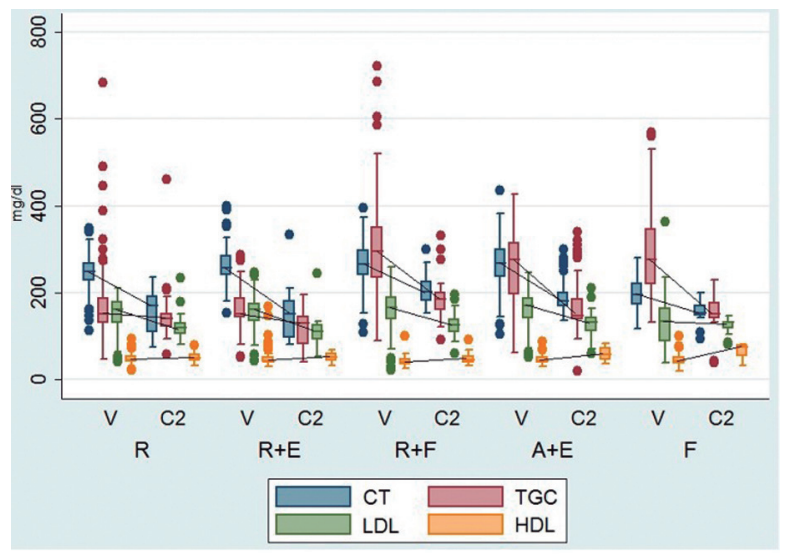

V: Vinculación, C2: Segundo control, R: Rosuvastatina, E: Ezetimive, A:Atorvastatina, F: Fibrato, CT: Colesterol total, TGC: Trigliceridos, LDL: Lipoproteinas de baja densidad, HDL: Lipoproteinas CT: Colesterol to:
de alta densidad

Figura 1. Mediciones del perfil lipídico según la terapia hipolipemiante recibida en la visita inicial y el segundo control. V: valores basales en la primera visita, C2: valores en el segundo control, R: Rosuvastatina, E: Ezetimibe, F: Fenofibrato, A: Atorvastatina, CT: Colesterol total, TGC: Triglicéridos

Fuente: Autores

Con respecto a la prescripción del tratamiento farmacológico, se compararon los valores basales del perfil lipídico entre el grupo que recibió monoterapia y el de terapia combinada, con los siguientes resultados: CT en monoterapia 228.5 (197.5-258) (med, RIQ) vs. en combinación 260 (238-298) $(\mathrm{p}=0.000)$, TGC en monoterapia 184 (145.5-263.5), vs. combinación 232 (158-300) ( $p=0.001)$, LDL en monoterapia 152.2 (112174), vs. combinación $166(139-186)(p=0.000)$ y HDL en monoterapia 45 (38-50), vs. combinación 40 (3849) $(p=0.06)$. Adicionalmente, se analizó la proporción de pacientes categorizados por grupos etarios según el tratamiento combinado o monoterapia, la cual se detalla en la tabla (Ver Tabla 3).

Con respecto a la frecuencia de eventos adversos relacionados con alguno de los medicamentos utilizados, no se evidenció ningún reporte en los registros incluidos en la plataforma, los cuales incluyeron los formatos referidos por el INVIMA.

\section{Discusión}

La utilización de registros biomédicos es una alternativa para el seguimiento en la utilización de medicamentos en vida real, lo cual constituye una herramienta que desde el punto de vista 
epidemiológico, permite desarrollar investigación ligada al proceso de atención y sus desenlaces con información en tiempo real, adicionalmente, se puede esperar que, a partir de un registro se mejoren los procesos y la calidad de la atención de los pacientes. Actualmente, algunos autores discuten la utilidad de los ensayos clínicos frente a la estimación real de la eficacia y efectividad de los fármacos, y aunque es claro que este tipo de diseño es capaz de minimizar la presencia de sesgos en condiciones ideales, las observaciones no son congruentes con lo que ocurre en la vida real de los pacientes que son tratados con moléculas que han demostrado eficacia ${ }^{19,20}$.

En el presente artículo se presentan resultados acerca del desempeño clínico y seguridad en la utilización de un grupo de medicamentos hipolipemiantes de una casa farmacéutica en Colombia. Se observó que los medicamentos hipolipemiantes tuvieron un impacto favorable sobre el perfil lipídico en la población estudiada.

Tabla 2. Valores de perfil lipídico para medicamentos discriminados según el tipo de combinación en las tres mediciones.

\begin{tabular}{|c|c|c|c|c|}
\hline & Vinculación (T0) & Control 1 (T1) & Control 2 (T2) & \\
\hline & \multicolumn{3}{|c|}{ Mediana (RIQ †) mg/dl } & Valor $p^{*}$ \\
\hline Estatinas & $(n=153)$ & $(n=120)$ & $(n=54)$ & \\
\hline Colesterol total & 249 (226-268) & $190(177.6-210)$ & $170(108-170)$ & 0 \\
\hline Triglicéridos & $150(129-186)$ & $145(120-154.5)$ & $139.5(120-139.5)$ & 0 \\
\hline LDL & $160(130-177)$ & $130(115-152-5)$ & $119.2(104-119.2)$ & 0 \\
\hline HDL & $45(40-52)$ & $49.5(42-52)$ & $49(43-49)$ & 0,01 \\
\hline Rosuvastatina + ezetimibe & $(n=76)$ & $(n=46)$ & $(n=29)$ & \\
\hline Colesterol total & $256(240-285)$ & $178(150-200)$ & $150(95-80)$ & 0 \\
\hline Triglicéridos & $150(142-187)$ & $138(100-145)$ & $130(80-144)$ & 0 \\
\hline LDL & $162(134-173)$ & $118(100-130)$ & $110(90-124)$ & 0 \\
\hline HDL & $40(40-50)$ & $46.5(42-55)$ & $51(42-60)$ & 0,01 \\
\hline Atorvastatina + ezetimibe & $(n=104)$ & $(n=85)$ & $(n=67)$ & \\
\hline Colesterol total & $267.5(235.5-300)$ & 210 (188-229) & $180(167-200)$ & 0 \\
\hline Triglicéridos & $276.5(196-313.5)$ & $179(148-220)$ & $147(138-184)$ & 0 \\
\hline LDL & 169 (140.5-187.5) & $141(122-151)$ & $131(110.3-141)$ & 0 \\
\hline HDL & $41(38-50)$ & $52(43-63)$ & $56(45-72)$ & 0 \\
\hline Fibrato & $(n=99)$ & $(n=74)$ & $(n=24)$ & \\
\hline Colesterol total & $195(172-220)$ & $178.5(160-190)$ & $151.5(146-151.5)$ & 0 \\
\hline Triglicéridos & $275(219-346)$ & 201 (172-239) & $150.5(140-150.5)$ & 0 \\
\hline LDL & $134(86-163)$ & $128(102-145)$ & $130.5(117-130.5)$ & 0,002 \\
\hline HDL & $42(36-50)$ & $48.5(45-60)$ & $73(52-73)$ & 0,001 \\
\hline Fibrato + rosuvastatina & $(n=68)$ & $(n=50)$ & $(n=24)$ & \\
\hline Colesterol total & $264.5(239-297.5)$ & $211(188-250)$ & $201(180-201)$ & 0 \\
\hline Triglicéridos & $295(233-350)$ & $211.5(156-250)$ & $185(159-185)$ & 0 \\
\hline LDL & 165 (137.4-189) & $145.5(112-158)$ & $125(108-125)$ & 0,009 \\
\hline $\mathrm{HDL}$ & $40(34-46.5)$ & $45(37-50)$ & $45(38.5-45)$ & 0,037 \\
\hline
\end{tabular}

† Rango intercuartil. * Prueba estadística de Wilcoxon, comparación de T0 vs. T2.

Fuente: Autores.

En el caso de la rosuvastatina sola, la disminución en el colesterol LDL fue cercana al $25 \%$ para la segunda medición, datos por debajo de los porcentajes de reducción esperadas que son mayores del 30\%21. Para el caso de la combinación atorvastatina + ezetemibe la reducción lograda del LDL fue del $45 \%$, lo cual está en los valores esperados de acuerdo con la tabla 2 (Ver Tabla 2) 22. Para el caso de los triglicéridos, cuando 
el paciente recibió fenofibrato solo, la reducción fue aproximadamente del $50 \%$ y en el caso de la combinación de fenofibrato con estatina la reducción fue de $38 \%$, aunque hubo disminución concomitante de LDL, por ende, de CT. La mayoría de los pacientes lograron meta de triglicéridos, menor de $200 \mathrm{mg} / \mathrm{dL}$. Estas reducciones fueron incluso mayores que las vistas en el estudio más grande que ha evaluado el fenofibrato a largo plazo, aunque en este último caso, el estudio se realizó sobre una población específica de pacientes diabéticos ${ }^{23}$. El impacto del fenofibrato solo también fue clínicamente importante sobre el $\mathrm{HDL}$, diferente a la combinación de fenofibrato y estatina, lo cual no tiene una explicación evidente.

En Colombia se identificó un estudio que evaluó la

Tabla 3. Utilización de monoterapia según edad.

\begin{tabular}{|c|c|c|}
\hline & \multicolumn{1}{|c|}{$\begin{array}{c}\text { Monoterapia } \\
\text { (n=252) }\end{array}$} & $\begin{array}{c}\text { Combinación } \\
\text { (n=249) }\end{array}$ \\
\cline { 2 - 3 } & $\mathbf{n}$ (\%) & $\mathbf{n ~ ( \% )}$ \\
\hline \multicolumn{3}{|c|}{ Edad } \\
\hline $\begin{array}{c}\text { Menores de } \\
30\end{array}$ & $12(70.6)$ & $5(29.4)$ \\
\hline $31-40$ & $23(56.1)$ & $18(43.9)$ \\
\hline $41-50$ & $44(46.8)$ & $50(53.2)$ \\
\hline $51-60$ & $78(51.3)$ & $74(48.7)$ \\
\hline $61-70$ & $51(50)$ & $50(49.5)$ \\
\hline $71-80$ & $37(48.7)$ & $39(51.3)$ \\
\hline $\begin{array}{c}\text { Mayores de } \\
80\end{array}$ & $7(35)$ & $13(65)$ \\
\hline
\end{tabular}

Fuente: Autores.

efectividad de hipolipemiantes en un grupo de 211 pacientes. En este, se observó una reducción del LDL de $21 \%$, un porcentaje un poco más bajo que el observado en esteestudio, aunqueaproximadamente el $20 \%$ de los pacientes utilizaron lovastatina, la cual es una estatina de menor potencia comparada con rosuvastatina o atorvastatina ${ }^{24-26}$.

Otro de los hallazgos importantes en el estudio fue la seguridad que ofrecieron las diversas combinaciones de hipolipemiantes. Los eventos adversos para este tipo de medicamentos individuales y en combinación, son reportados en menos de 3\% de los pacientes ${ }^{21,27}$. En el presente estudio, se tuvieron tres combinaciones de estatinas: fenofibratorosuvastatina, ezetimibe-rosuvastatina y ezetimibeatorvastatina, y no se reportaron eventos adversos relacionados en la historia clínica, aunque solo el
$39.5 \%$ tuvieron un segundo control después de la vinculación o prescripción del medicamento; y al ser eventos de baja prevalencia, es posible que el número de pacientes expuestos sea bajo. Las combinaciones evaluadas fueron tomadas a partir de la prescripción de los médicos incluidos en el presente estudio. Es importante anotar al respecto, que en el análisis estratificado por edad las combinaciones fueron recibidas por aproximadamente la mitad de los pacientes entre los 30 y 80 años, independiente del rango de edad. Esta conducta es muy particular en el contexto clínico en donde se realizó el estudio y no está en consonancia con las recomendaciones de las guías de manejo, a partir de la cual se espera que muchos pacientes reciban monoterapia con estatina como parte de su manejo inicial, ni con lo visto en otros estudios en donde la proporción de pacientes que reciben terapias combinadas es muy baja ${ }^{28,29}$.

Las reducciones del perfil lipídico que se dan progresivamente en este estudio están relacionadas con disminución en la incidencia de eventos cardiovasculares y cerebrovasculares, que la literatura estima en un $20 \%$, cuando se disminuye el LDL en $40 \mathrm{mg} / \mathrm{dl}$, metas logradas en los pacientes que utilizaron estatinas en monoterapia y combinadas (Ver Tabla 2). Esta indicación terapéutica hace parte de las "Guías de Práctica Clínica para la detección temprana, diagnóstico, tratamiento y seguimiento de las dislipidemias en la población mayor de 18 años" $(G P C)$, la cual recomienda determinar condiciones clínicas para iniciar y mantener manejo médico, entre las que se encuentran valores de LDL mayor de $160 \mathrm{mg} / \mathrm{dl}$, situación que fue característica en los pacientes evaluados ${ }^{14}$. Se observó en la vinculación, que en 148 (29.5\%) pacientes que tenían LDL elevados y TGC por debajo de $500 \mathrm{mg} / \mathrm{dl}$ iniciaron tratamiento farmacológico con fibratos en monoterapia o combinado, indicación que según las GPC no se recomienda como primera línea de tratamiento en esta población, sugiriendo una adherencia parcial por parte de los médicos generales a las GPC. Es importante mencionar que en este estudio no se involucró la evaluación del riesgo cardiovascular, ni la prescripción de actividad física o recomendaciones en la dieta, componentes importantes en la valoración y manejo integral de estos pacientes, por lo que no fue posible evaluar la adherencia de estos componentes de la GPC. La falta de adherencia por parte de los médicos a diversas recomendaciones establecidas en guías de manejo es un problema bien conocido en la práctica clínica, se han estudiado sus causas y también se ha demostrado que intervenciones 
múltiples de implementación de guías son los que aseguran que los porcentajes de adherencia mejoren, lo cual no es una práctica ampliamente utilizada en nuestro medio, en el contexto de la medicina general. Teniendo en cuenta lo anterior, las GPC establece una sección especial donde plantea precisamente cuáles son los elementos de implementación de la guía que se deben tener en cuenta para facilitar la adherencia de esta ${ }^{14}$.

Dentro de las limitaciones, encontramos dificultades en el seguimiento de toda la cohorte, lo cual puede limitar el análisis de los datos existentes. No fue posible evaluar el grado de adherencia a los medicamentos, importante en temas de evaluación de eficacia y seguridad. Sin embargo, esto también es propio de los estudios de vida real y del ejercicio médico cotidiano.

\section{Conclusiones}

En el presente estudio, la administración de estatinas solas o en combinación disminuyó los niveles de LDL y CT, siendo mejor en la combinación de fenofibratos + rosuvastatina y atorvastatina + ezetimibe, mientras que el fenofibrato solo demostró su desempeño clínico al disminuir los triglicéridos. Con respecto a las indicaciones de la GPC y su adherencia, los médicos participantes parcialmente cumplen con las recomendaciones, pues en un porcentaje considerable se inicia tratamientos de segunda línea en patologías debutantes. No se reportaron efectos adversos con el uso de los medicamentos involucrados.

\section{Declaración de conflictos de interés}

Este estudio fue financiado por Abbott-Lafrancol Colombia. Los autores declaran que la recolección, el análisis de la información y la discusión del estudio fue independiente.

\section{Referencias bibliográficas}

1. Coloma PM, Trifiro G, Patadia V, Sturkenboom M. Postmarketing safety surveillance: where does signal detection using electronic healthcare records fit into the big picture? Drug Saf. 2013;36(3):183-97.

2. Ministerio de la Protección Social-Universidad Nacional de Colombia. Estudio sobre uso y prescripción de medicamentos en cinco ciudades colombianas. Bogotá; 2005.

3. Soto-Álvarez J. Estudios observacionales para evaluar la efectividad clínica de los medicamentos. Uso de listas-guía para su diseño, análisis e interpretación. Atención primaria. 2005;35(3):156-162.
4. Saladrigas MV, Sacristán JA. Fichas de MedTrad (n. 13): eficacia, efectividad y eficiencia en la investigación de fármacos. Panace@. 2004;5(17-18):188-190.

5. Calderón CA, Urbina A. La Farmacovigilancia en los últimos 10 años: actualización de conceptos y clasificaciones. Logros y retos para el futuro en Colombia. Med UIS. 2011; 24(1):53-66

6. Furberg CD, Pitt B. Withdrawal of cerivastatin from the world market. Curr Control Trials Cardiovasc Med. 2001; 2(5):205-7.

7. Epstein RA. Regulatory paternalism in the market for drugs: lessons from Vioxx and Celebrex. Yale J Health Policy Law Ethics. 2005; 5(2):741-70.

8. López-Jaramillo P, López J. Lecciones aprendidas de dos grandes estudios epidemiológicos de enfermedades cardio-cerebrovasculares en las que ha participado Colombia. Rev Colomb Cardiol. 2010; 17(5):195-200.

9. Martínez JL. Factores asociados a la mortalidad por enfermedades no transmisibles en Colombia 2008-2012. Biomédica. 2016; 36:535-46.

10. Aorca-Martinez G, López-Jaramillo P, Molina DI, Sotomayor A, Accini JL., García H, et al. El aporte colombo-ecuatoriano al estudio hopE-3 ya la prevención primaria cardiovascular. Acta Med Colomb. 2016; 41(3):60-64.

11. Schargrodsky H, Hernández R, Champagne BM, Silva H, Vinueza $\mathrm{R}$, Silva LC, et al. CARMELA: assessment of cardiovascular risk in seven Latin American cities. Am J Med. 2008; 121(1):58-65.

12. Miranda JJ, Herrera VM, Chirinos JA, Gómez LF, Perel P, Pichardo $\mathrm{R}$, et al. Major cardiovascular risk factors in Latin America: a comparison with the United States. The Latin American consortium of studies in obesity (LASO). PLoS One. 2013; 8(1): e54056.

13. Galvis Y, Barona J, Cardona J. Prevalencia de dislipidemias en una institución prestadora de servicios de salud de Medellín (Colombia), 2013. Rev CES Med. 2016; 30(1): 3-13.

14. Ministerio de Salud y Protección Social - Colciencias. Guía de práctica clínica para la prevención, detección temprana, diagnóstico, tratamiento y seguimiento de las dislipidemias en la población mayor de 18 años. 2014[enero, 2017], Guía 27. Disponible en: http:/gpc.minsalud.gov.co/gpc_sites/Repositorio/ Conv_563/GPC_dislipidemias/GPC_dislipidemias_profesionales. aspx.

15. Stone NJ, Robinson JG, Lichtenstein AH, Bairey Merz CN, Blum $\mathrm{CB}$, Eckel RH, et al. ACC/AHA guideline on the treatment of blood cholesterol to reduce atherosclerotic cardiovascular risk in adults: a report of the American College of Cardiology/American Heart Association Task Force on Practice Guidelines. J Am Coll Cardiol. 2014; 63(25): 2889-2934.

16. López-Jaramillo P, Camacho PA, Un reto para Colombia: mejorar la utilización de medicamentos probadamente beneficiosos en la prevención secundaria cardiovascular. Colombia médica. 2012; 43(1): 7-10.

17. Diaztagle JJ, Chaves WG, Sprockel JJ, Sastoque JA, Nieto JA, Barreto GA, Hernández JI. Cumplimiento de metas de lípidos, según guías ATP III en prevención secundaria en consulta externa de Medicina Interna. Acta Med Colomb. 2013; 38(2): 54-60.

18. Merchan A, Jaramillo C, Mendoza F, Agudelo JF. ¿Se están alcanzando las metas en el perfil lipídico de personas con enfermedad coronaria previa? Rev Colomb Cardiol 2011; 18(5): 262-267.

19. Bellows BK, Kuo KL, Biltaji E, Singhal M, Jiao T, Cheng Y, et al. Real-world evidence in pain research: a review of data sources. J Pain Palliat Care Pharmacother. 2014;28(3):294-304.

20. Lionetti G, Kimura Y, Schanberg LE, Beukelman T, Wallace CA, Ilowite NT, et al. Using registries to identify adverse events in rheumatic diseases. Pediatrics. 2013;132(5):e1384-94.

21. Catapano AL, Graham I, De Backer G, Wiklund O, Chapman MJ, Drexel H, et al. 2016 ESC/EAS Guidelines for the Management of Dyslipidemias. E Heart J. 2016; 37(39):2999-3058.

22. Machado JE, Moncada JC, Mesa G. Patrones de prescripción de antilipémicos en un grupo de pacientes colombianos. Rev Panam Salud Pública. 2008;23(3)179-87.

23. Ridker PM, Danielson E, Fonseca FA, Genest J, Gotto AM, Kastelein JJ, et al. Rosuvastatin to Prevent Vascular Events in Men and Women with Elevated C-Reactive Protein. N Engl J Med. 2008;359(21):2195-207.

24. Nicholls SJ, Ballantyne CM, Barter PJ, Chapman MJ, Erbel RM, Libby P, et al. Effect of two intensive statin regimens on progression of coronary disease. N Engl J Med. 2011;365(22):2078-87. 


\section{Diaztagle JJ, Castro CA, Buitrago DC}

25. Keech A, Simes RJ, Barter P, Best J, Scott R, Taskinen MR, et al. Effects of long-term fenofibrate therapy on cardiovascular events in 9795 people with type 2 diabetes mellitus (the FIELD study): randomised controlled trial. Lancet. 2005; 366:1849-1861.

26. Machado-Alba JE, Machado-Duque ME, Yepes MC, Manrique S, Tobón LM. Tratamiento hipolipemiante y su efectividad en pacientes de cuatro ciudades colombianas. Acta Med Colomb. 2016; 41: 181-186.

27. Vanegas A, Jaramillo N. Intolerancia a las estatinas, un reto en la
MÉD.UIS. 2019;32(1): 13-20

práctica clínica. Rev Colomb Cardiol. 2008; 15(2): 75-83.

28. Waters D, Brotons C, Chiang C, Ferrières J, Foody J, Jukema J, et al. Lipid treatment assessment project 2: a multinational survey to evaluate the proportion of patients achieving low-density lipoprotein cholesterol goals. Circulation 2009;120(1):28-34.

29. Boytsov S, Logunova N, Khomitskaya Y. Suboptimal control of lipid levels: results from the non interventional Centralized Pan Russian Survey of the Undertreatment of Hypercholesterolemia II (CEPHEUS II). Cardiovasc Diabetol. 2017; 16(1):158. 